\title{
Construction of a combinatorial library of chimeric tumor-specific promoters
}

\author{
Kirill N. Kashkin, Igor P. Chernov, Dmitry A. Didych, and Eugene D. Sverdlov \\ Shemyakin-Ovchinnikov Institute of Bioorganic Chemistry, Russian Academy of Sciences, Moscow, Russia
}

BioTechniques 63:107-116 (September 2017) doi 10.2144/000114586

Keywords: artificial promoter; chimeric promoter; cancer specific; random promoter library; cancer gene therapy

Supplementary material for this article is available at www.BioTechniques.com/article/114586.

Gene therapy is a fast-developing field of molecular medicine. New, effective, and cancer-specific promoters are in high demand by researchers seeking to treat cancer through expression of therapeutic genes. Here, we created a combinatorial library of tumor-specific chimeric promoter modules for identifying new promoters with desired functions. The library was constructed by randomly combining promoter fragments from eight human genes involved in cell proliferation control. The pool of chimeric promoters was inserted into a lentiviral expression vector upstream of the CopGFP reporter gene, transduced into A431 cells, and enriched for active promoters by cell sorting. The enriched library contained a remarkably high proportion of active and tumor-specific promoters. This approach to generating combinatorial libraries of chimeric promoters may serve as a useful tool for selecting highly specific and effective promoters for cancer research and gene therapy.

One prerequisite for successful genetherapy cancer treatment is an adequate system for therapeutic gene expression in cancer cells. Ideally, this system should provide tissue-specific and strong expression of the transgene to ensure both safety and efficacy. Currently, natural tissue- or tumor-specific promoters are used for this purpose (1-3). However, these promoters have two important drawbacks. First, they are, as a rule, considerably less active than strong constitutive promoters, such as the CMV and SV40 promoters. Second, most promoters that have been described are generally highly active only in certain cancer cells $(1,2,4)$. Even relatively strong tumor-specific promoters with a comparatively wide spectrum of activity, such as the phSurv promoter from the BIRC5 gene for the apoptosis inhibitor survivin (5-7) or the TERT gene promoter (phTERT) from the human telomerase catalytic subunit gene (8-10), are not active in all types of cancer, and their relative activity significantly varies between tumor cell lines. For instance, BIRC5 promoter activity varies from $0.06 \%-16 \%(7,11,12)$ to $10 \%-40 \%(13)$ of CMV promoter activity.

A number of approaches have been proposed to increase the efficiency of tumor-specific promoters. These can be subdivided into two groups:

1. Approaches using bimodal therapeutic gene expression systems (14-16). One such approach exploits transcriptional transactivators such as the Tat-TAR system from the human immunodeficiency virus (HIV) (14). In the binary system, the tat activator gene is expressed under the control of a cancer-specific promoter, whereas the therapeutic gene is expressed under the control of a TAR-containing non-specific promoter. Tat protein binding to the TAR region of the non-specific promoter results in highlevel cancer-specific expression of the therapeutic gene. Another system utilizes the $\mathrm{P} 1$ bacteriophage Cre recombinase (17). Here, one vector harbors the Cre recombinase gene under the control of a tumor-specific promoter, while the other vector carries a therapeutic gene separated from a constitutive promoter by a polyadenylation signal flanked with two loxP sequences in direct orientation. When both vectors are transfected into a tumor cell, Cre recombinase excises the polyadenylation signal, which triggers high-level cancer-specific expression of the therapeutic gene.

2. Approaches using tumor-specific hybrid, double, or tandem promoters. An example of one of these promoters is the chimeric promoter comprising phTERT and a minimal cytomegalovirus promoter (phTERT-CMV) (18). This promoter was reported to be tumor-specific and more

\section{METHOD SUMMARY}

A combinatorial library of chimeric promoters was constructed by random ligation of a mixture of restriction fragments from known tumor-specific and constitutive human promoters. Chimeric promoters were then cloned into a lentiviral expression vector upstream of the CopGFP reporter gene and transduced into A431 cells. The library was enriched for active promoters by selection of fluorescent CopGFP-positive cells using a cell sorter, and selected promoters were then assayed for functional activity. 
active than non-modified phTERT. Using double tumor-specific promoters of the $h A S H 1$ and EZH2 genes, Poulsen at al. achieved efficient expression of a suicide gene in small cell lung cancer (SCLC) cells (19). The activity of the double promoter exceeded that of the hASH promoter alone up to 8-fold while retaining specificity to SCLC cells. Alekseenko et al. constructed an hTERT- hSurv269 double promoter that was more active in most cancer cells and had lower activity in normal fibroblasts than each constituent promoter of the tandem (20). High-level expression of the tBid apoptosis activator in breast cancer cells was achieved with a hybrid promoter composed of phSurv and the promoter of the mucin gene, which is known to be expressed at an elevated level in mammary gland tumor cells (21).

Since these tumor-specific double promoters are highly active only in certain types of cancer, it would be desirable to develop universal cancerspecific promoters that are active in many different (if not in all) tumor types, and therefore suitable for universal gene therapeutic protocols.
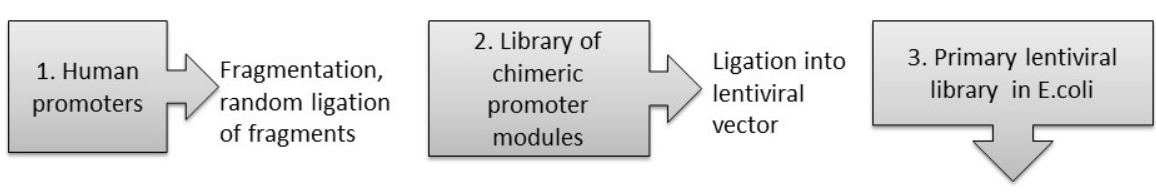

Packaging in 293T cells, isolation of virions

Alignment of chimeric and starting promoters. Identification of functionally important regions.
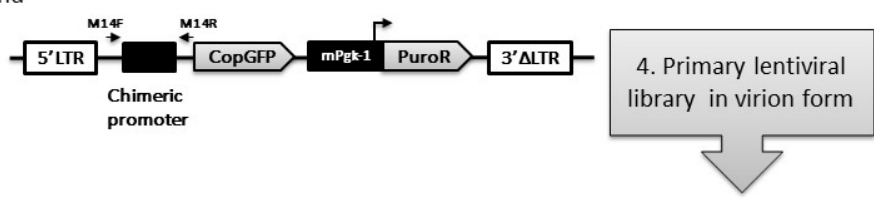

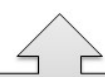

7. Individual cancer specific chimeric promoters

Sequence analysis of random clones, recloning into pGL3, luciferase test in cancer and normal cells
6. Enriched library of prospective promoters in PAL-2T

Figure 1. Flowchart of the chimeric promoter library construction. A fragment of lentiviral vector pLVPGm.1 with an inserted promoter module is presented in the schematic in the center. CopGFP: the green fluorescent protein reporter gene; mPgk-1: the mouse Pgk-1 promoter; PuroR: the puromycin resistance gene.

We hypothesized that a library consisting of random combinations of fragments from strong and tumorspecific promoters might contain chimeric promoters possessing enhanced therapeutic potential. In this work, we demonstrate the possibility of such an approach by constructing and analyzing a tumor
Transduction of A431 cells G418 selection, cell sorting, selection of luminous cells

Amplification, recloning into pAL-2T
5. Fraction of cells with prospective promoter modules

\section{. \\ Cyclic Dinucleotide STING Ligands}

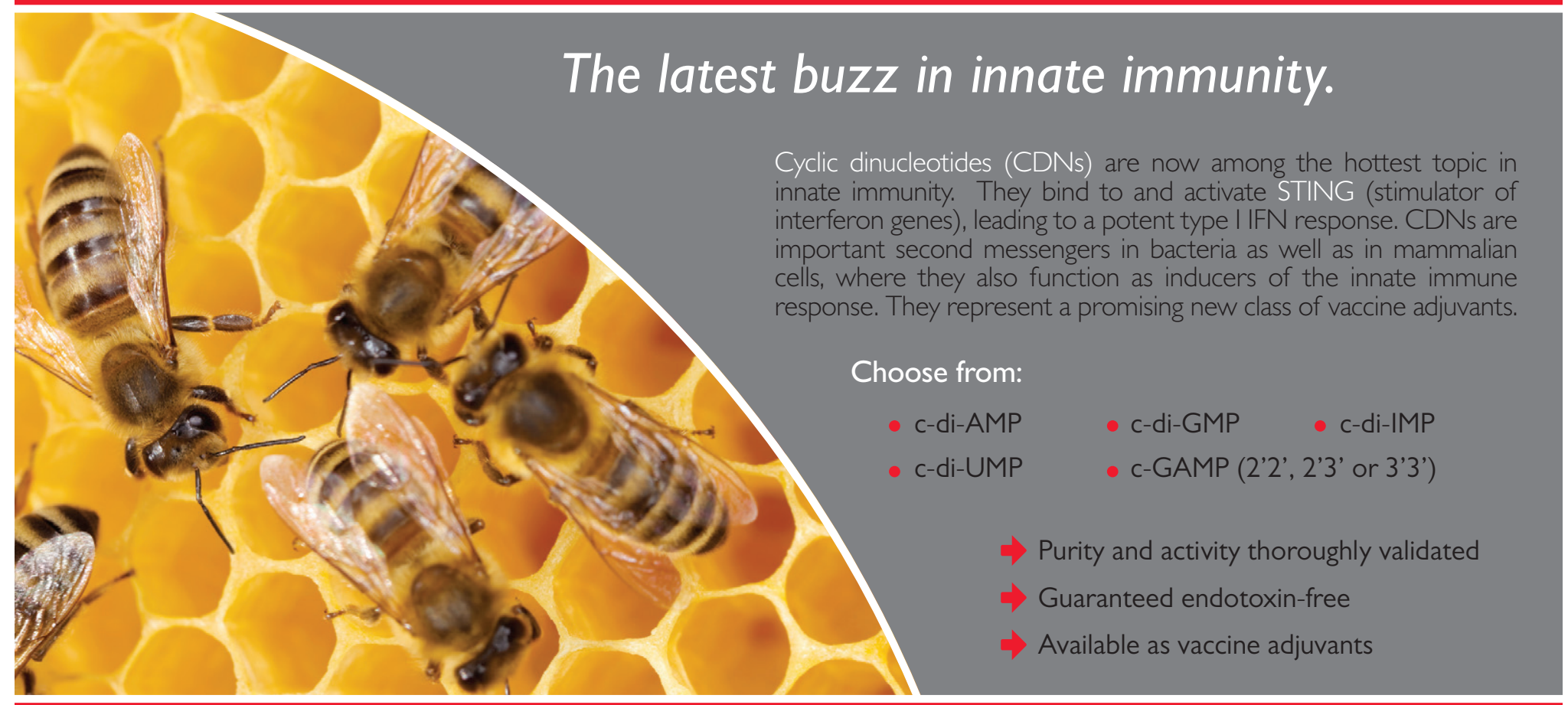


promoter-specific combinatorial library. Promoters in this library contained various combinations of randomly ligated fragments of eight human promoters involved in control of cell proliferation. Promoters of the CDC6, POLD1, CKS1B, MCM2, PLK1, BIRC5, TERT, and $P C N A$ genes, which were studied in our lab earlier $(12,20,22)$, were used in the construction of this library. All promoters except the PCNA promoter were shown to be tumor-specific but rather weak $(12,20)$, whereas the PCNA promoter is relatively strong (up to 30-fold compared with an SV40 promoter) and active in both normal and tumor cells of different origins (22).

\section{Materials and methods}

Vectors and cells

Control plasmids pGL3 Basic Vector, pGL3 Promoter Vector, and pRL-TK were purchased from Promega Corp. (Madison, WI,). Self-inactivating lentiviral expression vector pLVPGm.1 with the CopGFP fluorescent reporter gene (Evrogen, Moscow, Russia) was constructed from pLVT-MCSmut vector kindly provided by Dr. V.V. Belousov (Shemyakin and Ovchinnikov Institute of Bioorganic Chemistry RAS, Moscow, Russia). A431 cells (epidermoid carcinoma of skin), HT1080 cells (fibrosarcoma), and HEK 293T cells (human embryonic kidney cells stably expressing the SV40 large T antigen) were purchased from ATCC (Manassas, VA). ICL-7NS fibroblasts were prepared from normal lung tissue adjacent to a tumor according to the standard protocol $(23,24)$.

\section{Promoters}

Promoters from the CDC6, POLD1, CKS1B, MCM2, PLK1, and PCNA genes were amplified from PAL-TAbased plasmid clones using Pfu DNA polymerase (Thermo Fisher Scientific, Inc.; Waltham, MA) as described earlier $(12,22)$. All primers and PCR protocols are presented in Supplementary Table S1 and the Supplementary Materials and Methods. The human TERT promoter (phTERT) was isolated as a 271-bp Hindlll restriction fragment from the PhSTpGL3 plasmid (20), and the ends of the promoter were filled in with the Klenow fragment of Escherichia coli DNA polymerase I (Thermo Fisher Scientific). The BIRC5 269-bp promoter (PhSurv269) was amplified, using Pfu polymerase, from the PhSTpGL3 plasmid (20), kindly provided by Dr. M. Kostina (Shemyakin and Ovchinnikov Institute of Bioorganic Chemistry RAS, Moscow, Russia). All promoters were treated with bacteriophage T4 polynucleotide kinase (Thermo Fisher Scientific) and purified on silica membrane DNA cleanup columns (Cytokine, Saint-Petersburg, Russia) according to the manufacturer's instructions.

Chimeric promoter library construction Each promoter (4.5 pmol) was digested with BsuRI, and another 4.5 pmol was digested with Bsh1236l (both enzymes purchased from Thermo Fisher Scientific). Restriction endonucleases were then inactivated by heating, and the promoter digests were mixed together. One-tenth of the mix was self-ligated using T4
DNA ligase (Thermo Fisher Scientific). Ligation products were fractionated by electrophoresis on a $1 \%$ agarose gel, and 100-1000 bp DNA fragments were excised from the gel and purified using silica membrane mini columns from a Cleanup Mini Columns (Evrogen) according to manufacturer's instructions. Detailed instructions are provided in the Supplementary Material.

Cloning of the chimeric promoter library The resulting chimeric promoter library was ligated into the pLVPGm.1 plasmid that was digested with Eco32I restriction endonuclease (Thermo Fisher Scientific). The products of the reaction were purified using silica Cleanup Mini Columns (Evrogen) columns. Electroporation of the cloned promoter library in E. coli $\mathrm{DH} 5 \alpha$ competent cells was performed using the Bio-Rad Gene Pulser Xcell Electroporation System (Bio-Rad Inc., Hercules, CA) according to the manufacturer's protocol, and cells were plated onto 100-mm Petri dishes containing LB agar with $0.1 \mathrm{mg} / \mathrm{mL}$ ampicillin. Random clones from the library were tested for inserts by PCR using a Screenmix kit (Evrogen) with M14 primers (Supplementary Table S1) and by DNA sequencing of the PCR products.

\section{Lentiviral library selection}

The promoter library cloned in the pLVPGm.1 vector was transfected, along with a mixture of auxiliary plasmids [pMD.G (25), pCDNA3.1 puro-Nodomura B2 (26), and pCMV-dR8.91 (27)], into HEK 293T cells using Lipofectamine 2000 (Invitrogen, Carlsbad, CA)
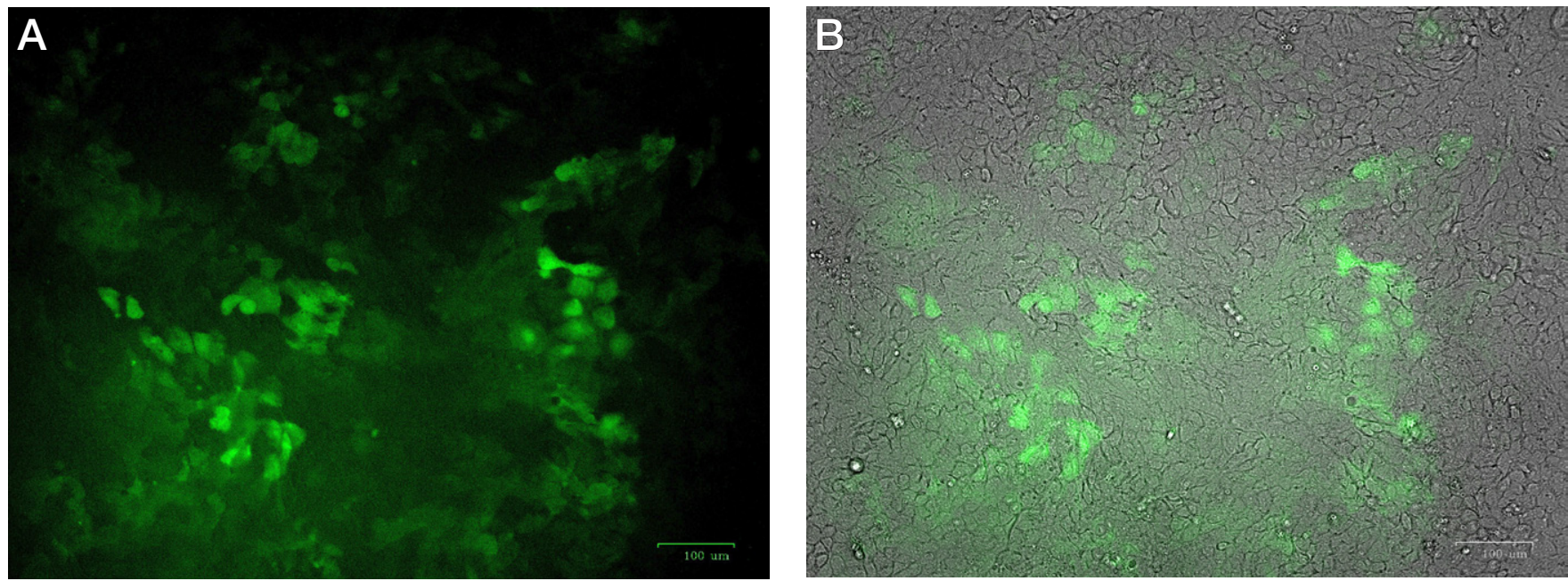

Figure 2. Microscopy of A431 cells infected by the lentiviral promoter library after cell sorting. (A) Fluorescence microscopy image. (B) Fluorescence microscopy image merged with phase-contrast microscopy image. 
Table 1. Promoter regions found in sequenced clones of the enriched library.

\begin{tabular}{|c|c|c|}
\hline Promoter & Fragment size (bp to TSS) & Times found ( $\mathbf{4})$ \\
\hline CDC6 & $125(-174 ;-50)$ & 7 \\
\hline PCNA & $90 \mathrm{bp}(-168 ;-79)$ & 4 \\
& $220 \mathrm{bp}(-129 ;+191)$ & 4 \\
\hline CKS1B & $177 \mathrm{bp}(-153 ;+24)$ & 4 \\
\hline MCM2 & $105 \mathrm{bp}(-227 ;-123)$ & 4 \\
\hline \multicolumn{2}{|l|}{} \\
\hline
\end{tabular}

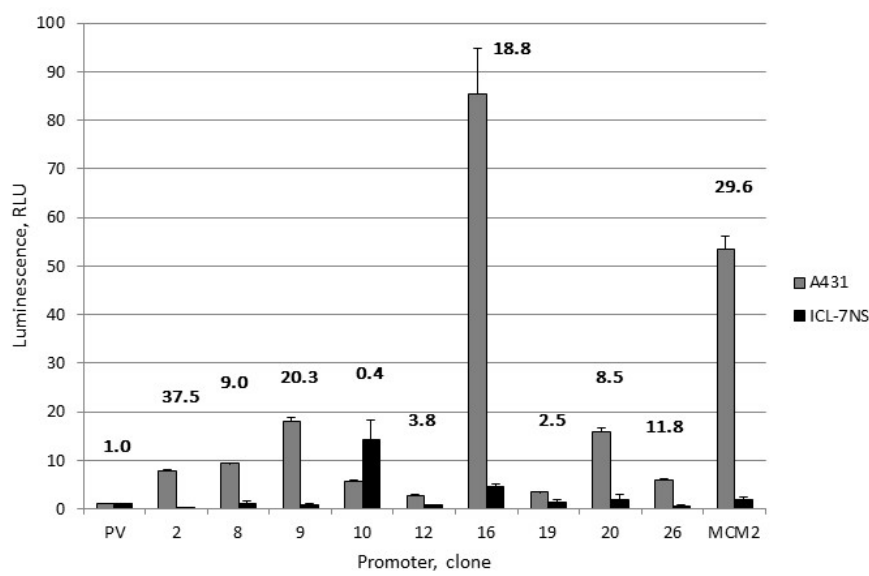

Figure 3. Chemiluminescence of A431 cells and normal ICL-7NS fibroblasts transfected with pGL3-based plasmids containing chimeric promoter modules. See Supplementary Table S2 for module structures. All data are normalized to SV40 promoter activity (designated as PV). MCM2 : the original MCM2 promoter. Results of four experiments with SEM are presented. A431/ICL-7NS ratios are denoted above the bars for each promoter.

according to the manufacturer's recommendations. Cells were incubated 18 $\mathrm{h}$ at $37^{\circ} \mathrm{C}$ and then $26 \mathrm{~h}$ at $32^{\circ} \mathrm{C}$. The cell culture media containing the lentivirus library was collected and stored at $-70^{\circ} \mathrm{C}$. Using $\mathrm{HT} 1080$ cells, the viral titer of the chimeric promoter library was estimated as $5 \times 10^{6}$ virions/milliliter (for details, see the Supplementary Material).

For selection of active chimeric promoters, $3.5 \times 10^{6} \mathrm{~A} 431$ cells were incubated for $24 \mathrm{~h}$ in a $\mathrm{CO}_{2}$-incubator at $37^{\circ} \mathrm{C}$, infected with the lentiviral promoter library (1.5 $\times 10^{7}$ virions), and incubated for an additional $48 \mathrm{~h}$. Cells were collected and sorted on a BD FACSAria III Flow Cytometer system (BD Biosciences, San Jose, CA) for fluorescent cells expressing the CopGFP protein. Approximately $1.5 \times 10^{5}$ of the selected cells were propagated for $72 \mathrm{~h}$. The cells were photographed using a ZOE Fluorescent Cell Imager (Bio-Rad) and then sedimented. Genomic DNA from the selected cells was isolated using a Qiagen QIAmp DNA Mini Kit (Qiagen, Hilden, Germany). The secondary library we obtained-presumably enriched with active promoter modules (the enriched promoter library) - was amplified using M14 primers. Amplification products were recloned into the $\mathrm{pAL}-2 \mathrm{~T}$ plasmid (Evrogen) using the AT-cloning procedure according to the manufacturer's recommendations, and random clones were isolated and sequenced. The fragment composition of individual promoter modules was determined using BLAST 2.2.10 (28) and the BioEdit (29) program. Promoter modules containing

\section{PCR just got easier ... and BETTER}

Good results are no longer enough to satisfy modern PCR product applications.

Simply add ThermaStop ${ }^{\mathrm{TM}}$ and ThermaGo $^{\mathrm{TM}}$ to your reaction and make your results GREAT.

\section{BETTER PCR Hot Start}

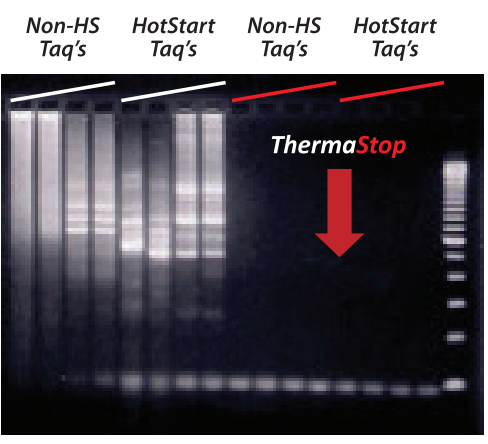

HS denotes a hot-start enzyme $1 \%$ Agarose : $1 \mathrm{~kb}$ Ladder

ThermaStop outperforms Hot Start Polymerases AND eliminates undesired product after cool down.

\section{BETTER specificity throughout PCR}

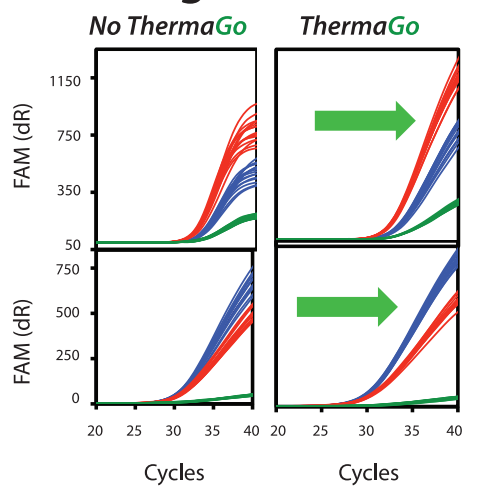

ThermaGo eliminates unwanted PCR errors, improves the production of correct products, AND increases assay reproducibility for end-point genotyping applications.
BETTER RT-PCR

Probe Signal

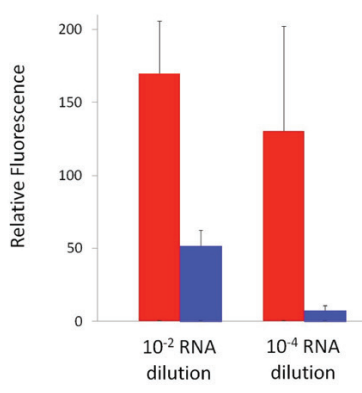

ThermaStop-for-RT improves sensitivity AND yield of one-step or two-step RT-PCR using gene-specific primers.

\section{You deserve the BEST results in your PCR assays - We are here to help!}


transcription start sites (TSSs) of any of the starting promoters were recloned in the pGL3 plasmid upstream of the luc gene in the same orientation as they were in pLVPGm.1 vector. TSSs were determined according to the sequences of mRNAs of the corresponding genes obtained from NCBI GenBank (Supplementary Table S1).

\section{Transfection of cells}

Transient transfections of cells were performed in duplicate in 24-well plates using Lipofectamine 2000 (Invitrogen), according to the manufacturer's recommendations. Twenty-four hour after transfection, cells were lysed, and the activities of Renilla and firefly luciferases were measured using a DualLuciferase Reporter Assay System (Promega) with a luminometer GENios Pro (Tecan, Männedorf, Switzerland). In all experiments, co-transfection with plasmid pRL-TK (Promega) was used as an internal control. Parallel transfections of the cells with pGL3 Basic Vector (pGL3-BV); pGL Promoter Vector (pGL3-PV) (Promega); pGL3-CMV Pr/ Enh Vector [pGL3-pCMV, containing an Asel/Bg/ll fragment of the CMV promoter of immediate early genes from the pEGFP-N1 plasmid (Clontech Laboratories, Inc., Mountain View, CA)]; as well as with the original MCM2 cloned in pGL3 (12); were performed in each experiment. The intensity values for firefly luciferase luminescence were normalized to those for Renilla luciferase, and data from duplicate wells were averaged. For each cell line under study, at least three independent transfections were performed. The data were corrected for background luminescence (pGL3-BV), normalized to the SV40 promoter activity (pGL3-PV), and then averaged. The resulting relative luminescence index was taken as a measure of the promoter activity in a given cell line. To estimate the cancer specificity of each promoter, its activity in A431 cells was additionally normalized to that in normal ICL-7NS fibroblasts.

Statistical analysis

Statistical analysis was performed using Prism 5 (GraphPad Software, Inc., La Jolla, CA), Statistica 8.0 (StatSoft, Inc., Tulsa, OK), and Microsoft Excel 2010 (Microsoft Corp., Redmond, WA).

\section{Results and discussion}

Combinatorial library construction

A flowchart for construction of a combinatorial library of chimeric promoters is presented in Figure 1. To prepare the library, cancer specific promoters CDC6 (803 bp), POLD1, (568bp), CKS1B, (334 bp), MCM2 (367 bp), PLK1 (439bp) (12), BIRC5 (269 bp), TERT, (243 bp) (20), and the strong universal PCNA promoter (389 bp) (22) were used. Promoters were fragmented by restriction digestion, and fragments were ligated together to generate random chimeric promoter combinations. The resulting library constituents could be easily analyzed by restriction digestion with the endonucleases used for their preparation. The library of chimeric promoter modules was size fractionated, the 100-1000 bp fraction was cloned into the selfinactivating pLPVGm.1 lentivirus vector (Figure 1), and the library was screened as described above.

PCR analysis of 20 random clones using M14 primers showed at least 19 contained inserts. DNA sequencing of two clones confirmed that they

\section{作

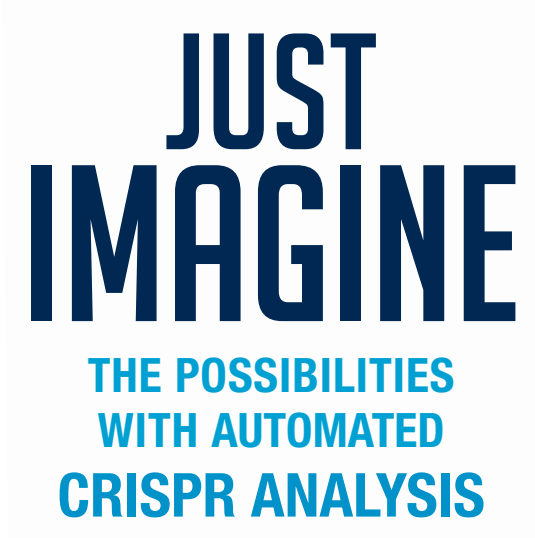

\section{contained chimeric promoter modules}

Fragment Analyzer ${ }^{\mathrm{TM}}$ is the only automated instrument for the analysis of CRISPR/Cas9 gene-editing events. Accelerate your scientific discovery using a streamlined process for easy identification of both individual and pooled gene mutations. 
consisting of fragments of the initial promoters (Supplementary Figure S1).

Library selection for active

promoter modules

The combinatorial promoter library in the lentiviral vector was converted into virions in HEK 293T cells. A431 cells were infected by the library as described above, sorted by flow cytometry, and fluorescent cells were collected (Figure 2).

Genomic DNA from these cells was isolated and amplified using M14 primers. Amplification products were cloned into the $\mathrm{pAL}-2 \mathrm{~T}$ plasmid, producing a plasmid library that presumably contained active promoter modules (referred to below as an "enriched library").

\section{Analysis of enriched library}

Inserts from 30 randomly selected clones from the enriched library were sequenced and aligned with the starting promoters. Twenty-six clones contained different fragment combinations from the starting promoters (Supplementary Table S2). Of these, 11 clones contained single fragments from individual promoters, some of which were inserted in the direction opposite to their normal orientation (promoter modules \#2, \#9, etc.). We suspect these fragments represent minimal promoters with bidirectional activity, since this would agree with known data on the bidirectionality of the PCNA, CKS1B and possibly POLD1 promoters (30), as well as with the known ability of eukaryotic promoters to initiate transcription in both directions (31). Other promoter modules included various combinations of fragments of the starting promoters.

Sequencing of 26 clones from the enriched library revealed that some promoter fragments were observed more often than others, likely due to their functionality. Examples of this include two regions from the PCNA promoter and one region each from the CDC6, CKS1B, and MCM2 promoters (Table 1, Supplementary Figure S2). It is noteworthy that fragments from the CDC6, PCNA, and CKS1B promoters in the enriched library were derived from promoter regions enriched in regulatory sites, as reported by other authors (32-35). A 105-bp fragment of the MCM2 promoter probably also has some crucial functions, but this promoter had not been studied in detail previously. Fragments from the POLD1 promoter projected onto the starting promoter sequence were distributed more uniformly, whereas fragments of the PLK1, BIRC5, and TERT promoters were rarely found in the sequenced chimeric modules.

\section{Functional tests of combinatorial} promoter modules

Following preliminary functional selection of the combinatorial library of chimeric promoter modules, we next directly assessed whether the selected promoter modules possess promoter activity. We hypothesized that promoter modules containing the TSS of any promoter (Supplementary Table S2) were more likely to be active than modules lacking a TSS. Therefore, we recloned nine promoter modules with the TSS from pAL-2T into the pGL3 plasmid upstream of the luc gene in the same orientation as in the lentiviral vector.

We transfected A431 cells with these clones and measured luciferase activity in the cell lysates (Figure 3 ). Parallel transfections with appropriate controls were performed, and data were normalized as described above.

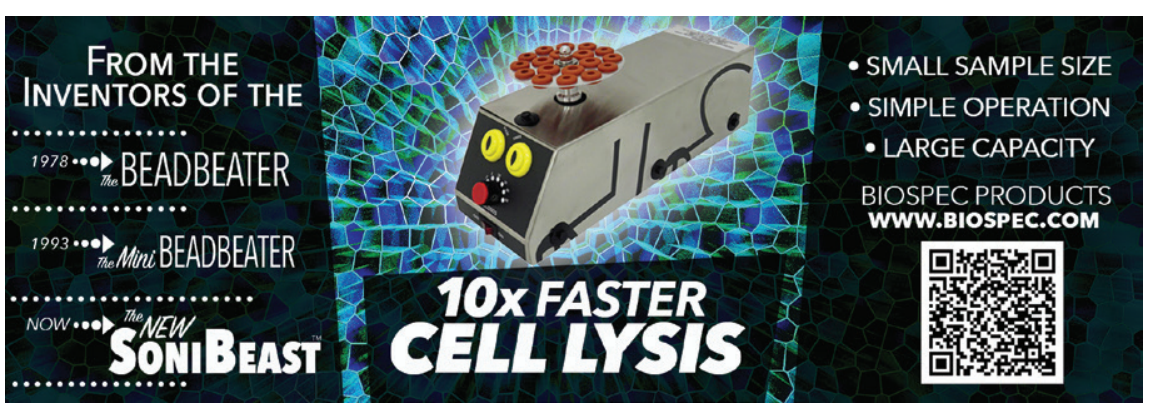

All 9 chimeric modules could drive luc gene expression to levels $\geq 2$.7-fold higher than the SV40 promoter (designated as PV in Figure 3). Among these, modules \#9, \#16, and \#20 were found to be the most active in A431 cells, being approximately 18-, 85- and 16-fold more active, respectively, than the SV40 promoter, whereas the original MCM2 promoter was 53-fold as active as the SV40 promoter. Thus, module \#16 was more effective than the MCM2 promoter and approximately as active as the PCNA promoter (22).

To study tumor specificity, we transfected normal ICL-7NS fibroblasts and A431 epidermoid carcinoma cells with the nine clones and compared luciferase activity in the transfected cells (Figure $3)$. With one exception (clone \#10), all chimeric promoters were more active in tumor cells than in normal fibroblasts. In some cases the difference was very prominent. For example, the activity of chimeric promoter \#16 was almost 20-fold higher in A431 cells than in normal fibroblasts. Another promoter module (clone \#2) was more specific for tumor cells than the MCM2 promoter, being 7.7-fold more active than the SV40 promoter. In contrast, promoter \#10 was 2-fold more active in normal fibroblasts than in A431 cells, a finding of special interest. To investigate the properties of each promoter in greater detail, it is necessary to test their activity in other tumor cells from different sources.

Here, we demonstrated that construction of random combinatorial libraries from fragmented native promoters is a useful approach in designing and selecting new, highly specific and effective promoters for cancer research and gene therapy. We suspect native tissue or cancer specific promoters are not as strong as ubiquitous or constitutive promoters because of the need for delicate gene regulation. However, therapeutic promoters, especially for cancer gene therapy, should be both specific and highly active. The approach here combines elements of strong constitutive promoters with highly tumorspecific promoters in an effort to obtain chimeric promoters of high strength and tumor specificity. Such promoters will be of considerable value for gene therapy applications. 


\section{Author contributions}

E.D.S. designed the study and performed general oversight of the work. K.N.K. and I.P.C. designed experimental approaches, performed the experiments, and critically reviewed the data. D.A.D. constructed the pLVPGm.1 vector that was used for cloning of the library of chimeric promoters. K.N.K. and E.D.S. wrote the manuscript.

\section{Acknowledgments}

This work was supported by the Russian Science Foundation (project No. 14-5000131). We thank Dr. Eugene P. Kopantzev for technical assistance and invaluable consultation regarding experiments and the analysis of the results of our study. We are grateful to Dr. Boris O. Glotov (Institute of Molecular Genetics, RAS) for critical reading of the manuscript.

\section{Competing interests}

The authors declare no competing interests.

\section{References}

1. Lee, S.E., R.J. Jin, S.G. Lee, S.J. Yoon, M.S. Park, D.S. Heo, and H. Choi. 2000. Development of a new plasmid vector with PSA-promoter and enhancer expressing tissuespecificity in prostate carcinoma cell lines. Anticancer Res. 20:417-422.

2. Yamamoto, M., R. Alemany, Y. Adachi, W.E. Grizzle, and D.T. Curiel. 2001. Characterization of the cyclooxygenase-2 promoter in an adenoviral vector and its application for the mitigation of toxicity in suicide gene therapy of gastrointestinal cancers. Mol. Ther. 3:385-394.

3. Van Houdt, W.J., Y.S. Haviv, B. Lu, M. Wang, A.A. Rivera, I.V. Ulasov, M.L. Lamfers, D. Rein, et al. 2006. The human survivin promoter: a novel transcriptional targeting strategy for treatment of glioma. J. Neurosurg. 104:583-592.

4. Adachi, Y., P.N. Reynolds, M. Yamamoto, M. Wang, K. Takayama, S. Matsubara, T. Muramatsu, and D.T. Curiel. 2001. A midkine promoter-based conditionally replicative adenovirus for treatment of pediatric solid tumors and bone marrow tumor purging. Cancer Res. 61:7882-7888.

5. Ambrosini, G., C. Adida, and D.C. Altieri. 1997. A novel anti-apoptosis gene, survivin, expressed in cancer and lymphoma. Nat. Med. 3:917-921.

6. Fukuda, S. and L.M. Pelus. 2006. Survivin, a cancer target with an emerging role in normal adult tissues. Mol. Cancer Ther. 5:1087-1098.

7. Zhu, Z.B., S.K. Makhija, B. Lu, M. Wang, L. Kaliberova, B. Liu, A.A. Rivera, D.M. Nettelbeck, et al. 2004. Transcriptional targeting of tumors with a novel tumor-specific survivin promoter. Cancer Gene Ther. 11:256262

8. Hsu, C.P., J. Miaw, J.Y. Hsia, S.E. Shai, and C.Y. Chen. 2003. Concordant expression of the telomerase-associated genes in non-small cell lung cancer. Eur. J. Surg. Oncol. 29:594-599.

9. Gu, J. and B. Fang. 2003. Telomerase promoterdriven cancer gene therapy. Cancer Biol. Ther. 2:S64-S70.

10. Cong, Y.S., W.E. Wright, and J.W. Shay. 2002. Human telomerase and its regulation. Microbiol. Mol. Biol. Rev. 66:407-425.

11. Konopka, K., C. Spain, A. Yen, N. Overlid, S. Gebremedhin, and N. Duzgunes. 2009. Correlation between the levels of survivin and survivin promoter-driven gene expression in cancer and non-cancer cells. Cell. Mol. Biol. Lett. 14:70-89.

12. Kashkin, K., I. Chernov, E. Stukacheva, G. Monastyrskaya, N. Uspenskaya, E. Kopantzev, and E. Sverdlov. 2015. Cancer specificity of promoters of the genes controlling cell proliferation. J. Cell. Biochem. 116:299-309.

13. Chen, J.S., J.C. Liu, L. Shen, K.M. Rau, H.P. Kuo, Y.M. Li, D. Shi, Y.C. Lee, et al. 2004. Cancer-specific activation of the survivin promoter and its potential use in gene therapy. Cancer Gene Ther. 11:740-747.

14. Mingaleeva, R.N., I.P. Chernov, E.P. Kopantsev, L.L. Zavalova, A.V. Sass, and E.D. Sverdlov. 2010. Comparative analysis of herpes simplex virus thymidine kinase gene expression potentiation via HIV-1 Tat-TAR-system and cancer-specific promoters in p53(+) and p53(-) cells. Mol. Biol. (Mosk.) 44:507-514.

15. Ueda, K., M. Iwahashi, M. Nakamori, M. Nakamura, I. Matsuura, H. Yamaue, and H. Tanimura. 2001. Carcinoembryonic antigenspecific suicide gene therapy of cytosine deaminase/5-fluorocytosine enhanced by the cre/loxP system in the orthotopic gastric carcinoma model. Cancer Res. 61:6158-6162.

16. Nagayama, Y., E. Nishihara, M. litaka, H. Namba, S. Yamashita, and M. Niwa. 1999. Enhanced efficacy of transcriptionally targeted suicide gene/prodrug therapy for thyroid carcinoma with the Cre-loxP system. Cancer Res. 59:3049-3052.

17. Kingsman, S.M. and A.J. Kingsman. 1996. The regulation of human immunodeficiency virus type-1 gene expression. Eur. J. Biochem. 240:491-507.

18. Takakura, M., S. Kyo, T. Kanaya, H. Hirano, J. Takeda, M. Yutsudo, and M. Inoue. 1999. Cloning of human telomerase catalytic subunit (hTERT) gene promoter and identification of proximal core promoter sequences essential for transcriptional activation in immortalized and cancer cells. Cancer Res. 59:551-557.

19. Farokhimanesh, S., F. Rahbarizadeh, M.J. Rasaee, A. Kamali, and B. Mashkani. 2010. Hybrid promoters directed tBid gene expression to breast cancer cells by transcriptional targeting. Biotechnol. Prog. 26:505-511.

20. Alekseenko, I.V., V.V. Pleshkan, E.P. Kopantzev, E.A. Stukacheva, I.P. Chernov, T.V. Vinogradova, and E.D. Sverdlov. 2012. Activity of the upstream component of tandem TERT/ survivin promoters depends on features of the downstream component. PLoS One 7:e46474.

21. Lu, B., S.K. Makhija, D.M. Nettelbeck, A.A. Rivera, M. Wang, S. Komarova, F. Zhou, M. Yamamoto, et al. 2005. Evaluation of tumorspecific promoter activities in melanoma. Gene Ther. 12:330-338.

22. Sverdlov, E.D., I.P. Chernov, K.N. Kashkin, et al. 2015. Universal promoter for expression of therapeutic genes in mammalian cells (Patent RU 2551784), Russia
23. Adams, R.L.P. 1980. Cell Culture for Biochemists. Elsevier/North-Holland Biomedical Press, Amsterdam, The Netherlands.

24. Kopantzev, E.P., N.A. Vayshlya, M.R Kopantseva, V.I. Egorov, M. Pikunov, M.V. Zinovyeva, T.V. Vinogradova, I.B. Zborovskaya, and E.D. Sverdlov. 2010. Cellular and molecular phenotypes of proliferating stromal cells from human carcinomas. Br. J. Cancer 102:1533-1540.

25. Naldini, L., U. Blomer, F.H. Gage, D. Trono, and I.M. Verma. 1996. Efficient transfer, integration, and sustained long-term expression of the transgene in adult rat brains injected with a lentiviral vector. Proc. Natl. Acad. Sci. USA 93:11382-11388.

26. Sullivan, C.S. and D. Ganem. 2005. A virusencoded inhibitor that blocks RNA interference in mammalian cells. J. Virol. 79:7371-7379.

27. Zufferey, R., D. Nagy, R.J. Mandel, L. Naldini, and D. Trono. 1997. Multiply attenuated lentiviral vector achieves efficient gene delivery in vivo. Nat. Biotechnol. 15:871-875.

28. Song, N., X. Zhu, L. Shi, J. An, Y. Wu, and J. Sang. 2009. Identification and functional analysis of a CDE/CHR element in the POLD1 promoter. Sci. China C Life Sci. 52:551-559.

29. Li, B. and M.Y. Lee. 2001. Transcriptional regulation of the human DNA polymerase delta catalytic subunit gene POLD1 by p53 tumor suppressor and Sp1. J. Biol. Chem. 276:2972929739.

30. Kashkin, K.N., I.P. Chernov, E.A. Stukacheva, E.P. Kopantzev, G.S. Monastyrskaya, N.Y. Uspenskaya, and E.D. Sverdlov. 2013. Cancer specificity of promoters of the genes involved in cell proliferation control. Acta Naturae. 5:79-83.

31. Orekhova, A.S. and P.M. Rubtsov. 2013 Bidirectional promoters in the transcription of Mamm. Genomes. Biochemistry (Mosc.) 78:335-341.

32. Hateboer, G., A. Wobst, B.O. Petersen, L. Le Cam, E. Vigo, C. Sardet, and K. Helin. 1998. Cell cycle-regulated expression of mammalian CDC6 is dependent on E2F. Mol. Cell. Biol. 18:6679-6697.

33. Morris, G.F. and M.B. Mathews. 1990 Analysis of the proliferating cell nuclear antigen promoter and its response to adenovirus early region 1. J. Biol. Chem. 265:16116-16125.

34. Tommasi, S. and G.P. Pfeifer. 1999. In vivo structure of two divergent promoters at the human PCNA locus. Synthesis of antisense RNA and $S$ phase-dependent binding of E2F complexes in intron 1. J. Biol. Chem. 274:2782927838.

35. Rother, K., Y.Y. Li, K. Tschop, R. Kirschner, G.A. Muller, J. Mossner, and K. Engeland. 2007. Expression of cyclin-dependent kinase subunit $1(\mathrm{Cks} 1)$ is regulated during the cell cycle by a CDE/CHR tandem element and is downregulated by $\mathrm{p} 53$ but not by $\mathrm{p} 63$ or p73. Cell Cycle 6:853-862.

\section{Received 27 April 2017; accepted 24 July 2017.}

Address correspondence to Kirill N Kashkin, Shemyakin-Ovchinnikiov Institute of Bioorganic Chemistry, Mikluho-Maklaya street 16/10, Moscow, 117997 Russian Federation. E-mail: kachkine@yandex.ru

To purchase reprints of this article, contact: biotechniques@fosterprinting.com 